\title{
EXISTENCE, STABILITY, AND COMPACTNESS IN THE $\alpha$-NORM FOR PARTIAL FUNCTIONAL DIFFERENTIAL EQUATIONS ${ }^{1}$
}

\author{
BY
}

\author{
C. C. TRAVIS AND G. F. WEBB
}

\begin{abstract}
The abstract ordinary functional differential equation $(a / d t) u(t)$ - $A u(t)+F\left(u_{t}\right), u_{0}=\phi$, is studied, where $-A$ is the infinitesimal generator of an analytic semigroup of linear operators and $F$ is continuous with respect to a fractional power of $A$.
\end{abstract}

1. Introduction. Our objective in this paper is to investigate the local existence, continuability, and stability properties of a class of partial differential equations with deviating arguments in terms involving spatial partial derivatives. As a model for this class one may take the equation

$$
\begin{aligned}
\frac{\partial W}{\partial t}(x, t) & =\frac{\partial^{2} W}{\partial x^{2}}(x, t)+f\left(t, W(x, t-r), \frac{\partial W}{\partial x}(x, t-r)\right), \\
W(0, t) & =W(\pi, t)=0, \quad 0 \leqslant x \leqslant \pi, \quad t \geqslant 0, \quad t \geqslant 0, \\
W(x, t) & =\phi(x, t), \quad 0 \leqslant x \leqslant \pi, \quad-r \leqslant t<0,
\end{aligned}
$$

where $f$ is a linear or nonlinear scalar-valued function, $r$ is a positive real number, and $\phi$ is a given initial function. This work continues the investigations of [1], [2]. In [2] global existence is established for the above equation by treating the problem as an abstract ordinary functional differential equation in a Banach space of the form

$$
\begin{gathered}
d u(t) / d t=-A u(t)+F\left(t, u_{t}\right), \quad t>0, \\
u_{0}=\phi,
\end{gathered}
$$

where $-A$ is the infinitesimal generator of an analytic semigroup of linear operators $T(t), t>0$, the domain of $F$ is the product of $[0, \infty)$ and an appropriately defined function space, and $F(t, \Psi)$ is autonomous, nonlinear, and Lipschitz continuous with respect to a fractional power of $A$ in the second variable. In the present study, we wish to establish local existence of solutions under the assumption that $F$ is only continuous with respect to a fractional power of $A$ in the second variable. However, as is well known for

Received by the editors December 9, 1975 and, in revised form, October 5, 1976.

AMS (MOS) subject classifications (1970). Primary 34G05, 35B35.

${ }^{1}$ Research support by NSF MCS 75-06332A01.

- American Mathematical Society 1978 
differential equations in a Banach space, the continuity of $F$ alone is not sufficient to assure local existence of solutions without further restrictions on the semigroup $T(t)$. For our purposes, it will suffice to assume that the semigroup $T(t)$ is compact for each $t>0$.

In what follows $X$ will denote a Banach space with norm $\|\cdot\|, r$ a positive real number, and if $u$ is a function with domain [ $\sigma-r, \sigma+b)$, then for any $t \in[\sigma, \sigma+b), u_{t}$ will denote the function with domain $[-r, 0]$ defined by $u_{t}(\theta)=u(t+\theta),-r \leqslant \theta \leqslant 0$.

2. Local existence of solutions. In this section, we shall establish local existence to the integrated version of the equation

$$
\begin{aligned}
d u(t) / d t+A u(t) & =F\left(t, u_{t}\right), \quad t>0, \\
u_{0} & =\phi,
\end{aligned}
$$

in the space $C_{\alpha}$. These results extend those in [2], where existence of solutions is established under the assumption that $F$ is Lipschitz continuous with respect to a fractional power of $A$. We shall make the following assumptions on the operator $A$ and the function $F$ :

(2.2) $A$ is a closed densely defined linear operator in a Banach space $X$ and - $A$ is the infinitesimal generator of an analytic semigroup $T(t), t>0$, in $X$ satisfying $\|T(t) x\|<M e^{\omega t}\|x\|$ for $t>0, x \in X$, and where $M$ and $\omega$ are real constants.

(2.3) For $0 \leqslant \alpha<1$, the fractional power $A^{\alpha}$ satisfies $\left\|A^{\alpha} T(t) x\right\|<$ $M_{\alpha} e^{\omega t} t^{-\alpha}\|x\|$ for $t>0$ and $x \in X$, where $M_{\alpha}$ is a real positive constant.

(2.4) $A^{-\alpha} \in B(X, X)$ so that $D\left(A^{\alpha}\right)$ is a Banach space when endowed with the norm $\|x\|_{\alpha}=\left\|A^{\alpha} x\right\|$ for $x \in D\left(A^{\alpha}\right)$. We shall denote this Banach space by $X_{\alpha}$. Also $A^{-\alpha}$ satisfies $\left\|(T(t)-I) A^{-\alpha}\right\|<N_{\alpha} t^{\alpha}$ for $t>0$, where $N_{\alpha}$ is a real positive constant.

(2.5) $T(t)$ is compact for each $t>0$.

(2.6) We shall denote by $C_{\alpha}$ the Banach space of continuous functions $C\left([-r, 0] ; X_{\alpha}\right)$ with the norm

$$
\|\phi\|_{C_{\alpha}}=\sup _{-r<\theta<0}\left\|A^{\alpha} \phi(\theta)\right\| .
$$

(2.7) $F: D \rightarrow X$ is continuous, where $D$ is an open set in $R \times C_{\alpha}$.

Proposition 2.1. Suppose (2.2)-(2.7) hold. For each $(\sigma, \phi) \in D$, there exist $\Upsilon_{\phi}>0$ and a continuous function $u:\left[\sigma-r, \sigma+\Upsilon_{\phi}\right) \rightarrow D\left(A^{\alpha}\right)$ satisfying

$$
\begin{gathered}
u(t)=T(t-\sigma) \phi(0)+\int_{\sigma}^{t} T(t-s) F\left(s, u_{s}\right) d s, \quad \sigma<t<\sigma+\Upsilon_{\phi}, \\
u_{\sigma}=\phi .
\end{gathered}
$$

Note. $u(t)$ is called a mild solution of the equation $d u(t) / d t+A u(t)=$ $F\left(t, u_{t}\right)$. 
Proof. For any real $\beta, \gamma>0$, let $I_{\beta}=\{t: 0<t<\beta\}, \beta_{\gamma}=\{\psi \in$ $\left.C_{\alpha}:\|\psi\|_{C_{\alpha}} \leqslant \gamma\right\}$. Let $\beta, \gamma$ be such that $(\sigma+t, \phi+\psi) \in D$ and $\| F(\sigma+t, \phi$ $+\psi) \|<N$ for $(t, \psi) \in I_{\beta} \times \beta_{\gamma}$. Suppose $\tilde{\phi} \in C\left([\sigma-r, \sigma+\beta] ; X_{\alpha}\right)$ is the function defined by $\tilde{\phi}_{\sigma}=\phi, \tilde{\phi}(\sigma+t)=T(t) \phi(0)$ for $t \in I_{\beta}$. Suppose $\bar{\gamma}<\gamma$, and choose $0<\Upsilon_{\phi}<\beta$ so that

$$
M_{\alpha} N \int_{0}^{\Upsilon_{\phi}} e^{\omega s_{s}}-\alpha d s \leqslant \bar{\gamma}
$$

and

$$
\left\|\tilde{\phi}_{\sigma+t}-\phi\right\|_{C_{a}}<\gamma-\bar{\gamma} \text { for } t \in I_{\Upsilon_{\phi}} .
$$

Let $K_{0}$ be the set defined by

$$
K_{0}=\left\{\eta \in C\left(\left[-r, \Upsilon_{\phi}\right] ; X_{\alpha}\right): \eta_{0}=0,\left\|\eta_{t}\right\|_{c_{\alpha}}<\bar{\gamma} \text { for } 0<t<\Upsilon_{\phi}\right\} .
$$

Then $\left\|F\left(\sigma+t, \eta_{t}+\tilde{\phi}_{\sigma+t}\right)\right\| \leqslant N$ for $0 \leqslant t \leqslant \Upsilon_{\phi}$ and $\eta \in K_{0}$, since $\| \eta_{t}+$ $\tilde{\phi}_{o+t}-\phi \|_{C_{\alpha}}<\bar{\gamma}+\gamma-\bar{\gamma}=\gamma$. Consider the transformation $S: K_{0} \rightarrow$ $C\left(\left[-r, \Upsilon_{\phi}\right] ; X_{\alpha}\right)$ defined by

$$
\begin{gathered}
(S \eta)(t)=\int_{0}^{t} T(t-s) F\left(\sigma+s, \tilde{\phi}_{\sigma+s}+\eta_{s}\right) d s, \quad 0<t \leqslant \Upsilon_{\phi}, \\
(S \eta)_{0}=0 .
\end{gathered}
$$

Finding the fixed points of $S$ in $K_{0}$ is equivalent to finding solutions of (2.8) in $K_{0}$ since such fixed points $\eta$ and solutions $u$ of (2.8) through $(\sigma, \phi)$ are related by $u_{\sigma+t}=\tilde{\phi}_{\sigma+t}+\eta_{t}$. Notice that $S: K_{0} \rightarrow K_{0}$ since if $\eta \in K_{0}$ we have that $(S \eta)_{0}=0$ and

$$
\begin{aligned}
\|(S \eta)(t)\|_{\alpha} & <\int_{0}^{t}\left\|A^{\alpha} T(t-s)\right\|\left\|F\left(\sigma+s, \tilde{\phi}_{\sigma+s}+\eta_{s}\right)\right\| d s \\
& <M_{\alpha} N \int_{0}^{\Upsilon_{\phi}} e^{\omega s} s^{-\alpha} d s<\bar{\gamma} .
\end{aligned}
$$

We will show that the set $\left\{(S \eta)(t): \eta \in K_{0}\right\}$ is equicontinuous. For $0 \leqslant t_{1}<$ $t_{2}<r_{\phi}$,

$$
\begin{aligned}
& \left\|(S \eta)\left(t_{1}\right)-(S \eta)\left(t_{2}\right)\right\|_{\alpha} \\
& =\| \int_{0}^{t_{1}} A^{\alpha} T\left(t_{1}-s\right) F\left(\sigma+s, \tilde{\phi}_{\sigma+s}+\eta_{s}\right) d s \\
& \quad \quad-\int_{0}^{t_{2}} A^{\alpha} T\left(t_{2}-s\right) F\left(\sigma+s, \tilde{\phi}_{\alpha+s}+\eta_{s}\right) d s \| \\
& \quad<\left\|\int_{0}^{t_{1}} A^{\alpha}\left(T\left(t_{1}-s\right)-T\left(t_{2}-s\right)\right) F\left(\sigma+s, \tilde{\phi}_{\sigma+s}+\eta_{s}\right) d s\right\| \\
& \quad+\left\|\int_{t_{1}}^{t_{2}} A^{\alpha} T\left(t_{2}-s\right) F\left(\sigma+s, \tilde{\phi}_{\sigma+s}+\eta_{s}\right) d s\right\|
\end{aligned}
$$




$$
\begin{aligned}
& <\left\|\left(T(\varepsilon)-T\left(t_{2}-t_{1}+\varepsilon\right)\right) \int_{0}^{t_{1}-\varepsilon} A^{\alpha} T\left(t_{1}-s-\varepsilon\right) F\left(\sigma+s, \tilde{\phi}_{\sigma+s}+\eta_{s}\right) d s\right\| \\
& \quad+\left\|\int_{t_{1}-\varepsilon}^{t_{1}} A^{\alpha}\left(T\left(t_{1}-s\right)-T\left(t_{2}-s\right)\right) F\left(\sigma+s, \tilde{\phi}_{\sigma+s}+\eta_{s}\right) d s\right\| \\
& \quad+N M_{\alpha} \int_{0}^{t_{2}-t_{1}} e^{\omega s_{s}-\alpha} d s \\
& \leqslant\left\|T(\varepsilon)-T\left(t_{2}-t_{1}+\varepsilon\right)\right\| N M \int_{0}^{t_{1}-\varepsilon} e^{\omega s_{s}-\alpha} d s \\
& \quad+N M_{\alpha}\left[\int_{0}^{\varepsilon} e^{\omega s_{s}-\alpha} d s+\int_{0}^{t_{2}-t_{1}+\varepsilon} e^{\omega s_{s}-\alpha} d s\right] \\
& \quad+N M_{\alpha} \int_{0}^{t_{2}-t_{1}} e^{\omega s_{s}-\alpha} d s .
\end{aligned}
$$

The equicontinuity now follows from the fact that compactness of $T(t)$ for $t>0$ implies continuity of $T(t)$ for $t>0$ in the uniform operator topology.

Next, we show $\overline{S\left(K_{0}\right)}$ is compact. Since we have shown $S\left(K_{0}\right)$ is an equicontinuous collection, it suffices by the Arzela-Ascoli theorem to show $\left\{(S \eta)(t): \eta \in K_{0}\right\}$ is precompact in $X_{\alpha}$ for fixed $t \in\left[-r, \Upsilon_{\phi}\right]$. We shall accomplish this by showing that for $0<\alpha<\beta<1$ and for fixed $t \in$ $\left[-r, \Upsilon_{\phi}\right],\left\{\left(A^{\beta} S \eta\right)(t)\right\}$ is bounded in $X$ and appealing to the compactness of $A^{-\beta}: X \rightarrow X_{\alpha}$. Observe that

$$
\begin{aligned}
\left\|\left(A^{\beta} S \eta\right)(t)\right\| & =\left\|\int_{0}^{t} A^{\beta} T(t-s) F\left(\sigma+s, \tilde{\phi}_{\sigma+s}+\eta_{s}\right) d s\right\| \\
& <N M_{\beta} \int_{0}^{t} e^{\omega s_{s}-\beta} d s .
\end{aligned}
$$

Finally, we show that $S$ is continuous. Since $F: D \rightarrow X$ is continuous, given $\varepsilon>0$, there is a $\delta>0$, such that $\sup _{0<s<r_{+}}\|\eta(s)-\hat{\eta}(s)\|_{\alpha}<\delta$ implies that

$$
\sup _{0<s<r_{\phi}}\left\|F\left(\sigma+s, \tilde{\phi}_{\sigma+s}+\eta_{s}\right)-F\left(\sigma+s, \tilde{\phi}_{\sigma+s}+\hat{\eta}_{s}\right)\right\|<\varepsilon .
$$

Then, for $0<t<\Upsilon_{\phi}$ and $\sup _{0<s<r_{\phi}}\|\eta(s)-\hat{\eta}(s)\|_{\alpha}<\delta$,

$$
\begin{aligned}
& \|(S \eta)(t)-(S \hat{\eta})(t)\|_{\alpha} \\
& <M_{\alpha} \int_{0}^{t} e^{\omega(t-s)}(t-s)^{-\alpha} \| F\left(\sigma+s, \tilde{\phi}_{\sigma+s}+\eta_{s}\right) \\
& -F\left(\sigma+s, \tilde{\phi}_{\sigma+s}+\hat{\eta}_{s}\right) \| d s \\
& <\varepsilon M_{\alpha} \int_{0}^{t} e^{\omega s_{s}-\alpha} d s,
\end{aligned}
$$

and this yields the continuity of $S$. 
By Schauder's fixed point theorem, $S$ has a fixed point in $K_{0}$, and the proof of the theorem is complete.

We shall now investigate under what conditions the mild solutions of equation (2.1) are actually strong solutions. That is, we want to know conditions under which every solution $u(t)$ of the integral equation (2.8) is contained in $C\left(\left[\sigma-r, \sigma+\Upsilon_{\phi}\right) ; X_{\alpha}\right) \cap C^{1}\left(\left(\sigma, \sigma+\Upsilon_{\phi}\right) ; X\right)$.

Proposition 2.2. Suppose (2.2)-(2.7) hold and that $F: D \rightarrow X$ is locally Hölder continuous in both of its variables, i.e., in a neighborhood of any point in $D$, there exist constants $L$ and $0<\theta<1$ such that

$$
\|f(t, \eta)-F(\hat{t}, \hat{\eta})\|<L\left(\mid t-\hat{t}^{\theta}+\|\eta-\hat{\eta}\|_{c_{a}}^{\theta}\right),
$$

for all $(t, \eta),(\hat{t}, \hat{\eta})$ contained in the neighborhood. Then every mild solution of (2.1) is a strong solution.

Proof. If we show that the function $g(t)=F\left(t, u_{t}\right)$ is locally Hölder continuous on $\left(\sigma, \sigma+\Upsilon_{\phi}\right)$, then it follows from the theory of nonhomogeneous linear equations that $u$ is a strong solution [3]. That $g$ is locally Hölder continuous on $\left(\sigma, \sigma+\Upsilon_{\phi}\right)$ will follow if we prove that the map $t \rightarrow u(t)$ is locally Hölder continuous. Choose $\beta$ such that $\alpha<\beta<1$. Then

$$
\begin{aligned}
\|u(t)\|_{\beta} & <\left\|A^{\beta-\alpha} T(t-\sigma) A^{\alpha} \phi(0)\right\|+\left\|\int_{\sigma}^{t} A^{\beta} T(t-s) F\left(s, u_{s}\right) d s\right\| \\
& <M_{\beta-\alpha} e^{\omega(t-\sigma)}(t-\sigma)^{\alpha-\beta}\|\phi\|_{\alpha}+M_{\beta} \int_{\sigma}^{t} e^{\omega(t-s)}(t-s)^{-\beta}\left\|F\left(s, u_{s}\right)\right\| d s .
\end{aligned}
$$

Thus, $\|u(t)\|_{\beta}$ is bounded on any compact subset of $\left(\sigma, \sigma+\Upsilon_{\phi}\right)$. Now, for $\sigma<t<t+h<\sigma+\Upsilon_{\phi}$,

$$
u(t+h)-u(t)=(T(h)-I) u(t)+\int_{t}^{t+h} T(t+h-s) F\left(s, u_{s}\right) d s .
$$

Thus,

$$
\begin{aligned}
& \|u(t+h)-u(t)\|_{\alpha}<\left\|(T(h)-I) A^{-(\beta-\alpha)} A^{\beta} u(t)\right\| \\
& \quad+\left\|\int_{t}^{t+h} A^{\alpha} T(t+h-s) F\left(s, u_{s}\right) d s\right\| \\
& <N_{\beta-\alpha} h^{\beta-\alpha}\|u(t)\|_{\beta}+\sup _{t<s<t+h}\left\|F\left(s, u_{s}\right)\right\| M_{\alpha} \int_{0}^{h} e^{\omega s} s^{-\alpha} d s \\
& \quad<N_{\beta-\alpha}\|u(t)\|_{\beta} h^{\beta-\alpha}+\sup _{t<s<t+h}\left\|F\left(s, u_{s}\right)\right\|\left(\frac{M_{\alpha}}{1-\alpha}\right) \max \left\{1, e^{\omega T_{\odot}}\right\} h^{1-\alpha},
\end{aligned}
$$

which implies the local Hölder continuity on $\left(\sigma, \sigma+\Upsilon_{\phi}\right)$ of the map $t \rightarrow u(t)$. 


\section{Continuation of solutions.}

Proposition 3.1. Suppose (2.2)-(2.7) hold and that $F: D \rightarrow X$ takes closed bounded sets in $D$ into bounded sets in $X$. If $u$ is a noncontinuable solution of (2.1) existing on $[\sigma-r, b)$, then either $b=+\infty$, or given any closed bounded set $U$ in $D$, there exists a sequence $t_{k} \rightarrow b^{-}$such that $\left(t_{k}, u_{t_{k}}\right) \notin U$.

Proof. Assume that $b<+\infty$ and the conclusion of the theorem is false. Then there exists a closed bounded set $U$ in $D$ such that $\left(t, u_{t}\right) \in U$ for $\sigma<t<b$. Let $R=\sup f(t, \psi)$ for $(t, \psi) \in U$; then for $\sigma<t<t+h<b$ and $\alpha<\beta<1$,

$$
\begin{aligned}
\| u(t+h)- & u(t)\left\|_{\alpha} \leqslant\right\|(T(h)-I) A^{-(\beta-\alpha)} A^{\beta} u(t) \| \\
& +\left\|\int_{t}^{t+h} A^{\alpha} T(t+h-s) F\left(s, u_{s}\right) d s\right\| \\
< & N_{\beta-\alpha} h^{\beta-\alpha}\|u(t)\|_{\beta}+(R /(1-\alpha)) M_{\alpha} \max \left\{1, e^{\omega b}\right\} h^{1-\alpha} .
\end{aligned}
$$

Also, as we have seen before,

$$
\begin{aligned}
\|u(t)\|_{\beta} & <\left\|A^{\beta-\alpha} T(t-\sigma) A^{\alpha} \phi(0)\right\|+\left\|\int_{0}^{t} A^{\beta} T(t-s) F\left(s, u_{s}\right) d s\right\| \\
& <M_{\beta-\alpha} e^{\omega(t-\sigma)}(t-\sigma)^{\alpha-\beta}\|\phi\|_{\alpha}+M_{\beta} R \int_{0}^{t-\sigma} e^{\omega s_{s}-\beta} d s .
\end{aligned}
$$

Thus, $\|u(t)\|_{\beta}$ is bounded on compact subsets of $(\sigma, b)$. From this fact and equation (3.1) it follows that $u$ is uniformly continuous on $[\sigma-r, b)$. Hence $\lim _{t \rightarrow b}-u(t)$ exists and $u$ can be extended to a continuous function on $[\sigma-r, b]$. Since $\left(b, u_{b}\right) \in D$, one can find a solution of (2.1) through this point to the right of $b$. This contradicts the noncontinuability hypothesis on $u$ and proves the theorem.

Corollary 3.1. Suppose (2.2)-(2.7) hold and that $f:[\sigma, \infty) \times C_{\alpha} \rightarrow X$ is continuous and maps closed bounded sets in $[\sigma, \infty) \times C_{\alpha}$ into bounded sets in $X$. If $u$ is a noncontinuable solution of (1.0) existing on $[\sigma-r, b)$, then either $b=+\infty$ or $\varlimsup_{t \rightarrow b}-\left\|u_{t}\right\|_{c_{a}}=+\infty$.

4. Compactness of the semigroup of solutions. Throughout this section, we will suppose conditions (2.2)-(2.7) except that we require $F$ to be autonomous and Lipschitz continuous, that is $F: C_{\alpha} \rightarrow X$ and $\|F(\phi)-F(\psi)\|<\beta \| \phi-$ $\psi \|_{C_{\alpha}}$ for $\phi, \psi \in C_{\alpha}$, where $\beta$ is a positive constant. We also assume $F(0)=0$, which implies $\|F(\phi)\| \leqslant \beta\|\phi\|_{C_{\alpha}}$ for $\phi \in C_{\alpha}$. By virtue of Proposition 3.1 of [2], there exists for each $\phi \in C_{\alpha}$ a unique continuous function $u(\phi):[-r, \infty)$ $\rightarrow D\left(A^{\alpha}\right)$ satisfying 


$$
\begin{gathered}
u(\phi)(t)=T(t) \phi(0)+\int_{0}^{t} T(t-s) F\left(u_{s}(\phi)\right) d s, \quad t \geqslant 0, \\
u_{0}(\phi)=\phi .
\end{gathered}
$$

It follows from Proposition 3.2 of [2] that the solutions of (4.1) define a strongly continuous semigroup of nonlinear Lipschitz continuous operators $U(t), t \geqslant 0$, in $C_{\alpha}$ by $U(t) \phi=u_{t}(\phi)$. That is, the family $U(t), t \geqslant 0$, satisfies $U(t): C_{\alpha} \rightarrow C_{\alpha}$ for all $t \geqslant 0, U(0)=I, U(t+s)=U(t) U(s)$ for all $s, t \geqslant$ 0 , and $U(t) \phi$ is continuous from $[0, \infty)$ to $C_{\alpha}$ for each fixed $\phi \in C_{\alpha}$. Moreover, for $t \geqslant 0$ and $\phi, \psi \in C_{\alpha}$,

$$
\|U(t) \phi-U(t) \psi\|_{C_{\alpha}} \leqslant P(\delta) e^{\delta t}\|\phi-\psi\|_{C_{a}}
$$

where $\delta, P(\delta)$ are real constants.

Proposition 4.1 . The strongly continuous semigroup of nonlinear operators $U(t), t \geqslant 0$, is compact as a mapping in $C_{\alpha}$ for each fixed $t>r$.

Proof. Let $\left\{\phi_{\gamma}: \gamma \in \Gamma\right\}$ be a bounded subset of $C_{\alpha}$ and let $t>r$ be fixed, but arbitrary. Let $\|T(s)\| \leqslant M$ for $0 \leqslant s \leqslant t$, and recall (4.2) and the Lipschitz continuity of $F$ to argue that $\left\|F\left(u_{s}\left(\phi_{\gamma}\right)\right)\right\| \leqslant N$ for $0 \leqslant s \leqslant t$, $\gamma \in \Gamma$. For each $\gamma \in \Gamma$, define $f_{\gamma} \in C_{\alpha}$ by $f_{\gamma}=u_{t}\left(\phi_{\gamma}\right)$. Then

$$
f_{\gamma}(\theta)=T(t+\theta) \phi_{\gamma}(0)+\int_{0}^{t+\theta} T(t+\theta-s) F\left(u_{s}\left(\phi_{\gamma}\right)\right) d s
$$

for $-r<\theta<0$. We will first demonstrate that the family $\left\{f_{\gamma}: \gamma \in \Gamma\right\}$ is equicontinuous. Let $\gamma \in \Gamma, 0<\varepsilon<t-r,-r<\hat{\theta}<\theta<0$, and observe that

$$
\begin{aligned}
\| A^{\alpha} f_{\gamma}(\theta) & -A^{\alpha} f_{\gamma}(\hat{\theta}) \| \\
< & \left\|A^{\alpha} T(t+\theta) \phi_{\gamma}(0)-A^{\alpha} T(t+\hat{\theta}) \phi_{\gamma}(0)\right\| \\
& +\left\|(T(\varepsilon)-T(\theta-\hat{\theta}+\varepsilon)) \int_{0}^{t+\hat{\theta}-\varepsilon} A^{\alpha} T(t+\hat{\theta}-s-\varepsilon) F\left(u_{s}\left(\phi_{\gamma}\right)\right) d s\right\| \\
& +\left\|\int_{t+\hat{\theta}-\varepsilon}^{t+\hat{\theta}}\left[A^{\alpha} T(t+\hat{\theta}-s)-A^{\alpha} T(t+\theta-s)\right] F\left(u_{s}\left(\phi_{\gamma}\right)\right) d s\right\| \\
& +\left\|\int_{t+\hat{\theta}}^{t+\theta} A^{\alpha} T(t+\theta-s) F\left(u_{s}\left(\phi_{y}\right)\right) d s\right\| \\
< & \|T(t+\theta)-T(t+\hat{\theta})\|\left\|A^{\alpha} \phi_{\gamma}(0)\right\| \\
& +\|T(\varepsilon)-T(\theta-\hat{\theta}+\varepsilon)\| N M_{\alpha} \int_{0}^{t+\hat{\theta}-\varepsilon} e^{\omega s_{s}-\alpha} d s \\
& +N M_{\alpha}\left[\int_{0}^{e} e^{\omega s_{s}-\alpha} d s+\int_{0}^{\theta-\hat{\theta}+\varepsilon} e^{\omega s_{s}-\alpha} d s\right]+N M_{\alpha} \int_{0}^{\theta-\hat{\theta}} e^{\omega s_{s}-\alpha} d s .
\end{aligned}
$$


The equicontinuity now follows from the uniform continuity of $T(s), s \in$ $[\varepsilon, t]$, in $B(X, X)$.

We will now show that for fixed $\theta \in[-r, 0]$, the set $\left\{f_{\gamma}(\theta): \gamma \in \Gamma\right\}$ is precompact in $X_{\alpha}$. Choose $0<\beta$ such that $\alpha<\beta<1$ and observe that

$$
\begin{aligned}
\left\|A^{\beta} f_{\gamma}(\theta)\right\| \leqslant & \left\|A^{\beta-\alpha} T(t+\theta) A^{\alpha} \phi_{\gamma}(0)\right\| \\
& +\left\|\int_{0}^{t+\theta} A^{\beta} T(t+\theta-s) F\left(u_{s}\left(\phi_{\gamma}\right)\right) d s\right\| \\
< & \max \left\{1, e^{\omega(t+\theta)}\right\}(t+\theta)^{\alpha-\beta}\left\|\phi_{\gamma}\right\|_{C_{\varepsilon}} \\
& +N M_{\beta} \int_{0}^{t+\theta} e^{\omega s_{s}-\beta} d s .
\end{aligned}
$$

Thus $\left\{A^{\beta} f_{\gamma}(\theta): \gamma \in \Gamma\right\}$ is a bounded subset of $X$. The precompactness of the set $\left\{f_{\gamma}(\theta): \gamma \in \Gamma\right\}$ in $X_{\alpha}$ now follows from the compactness of $A^{-\beta}: X \rightarrow X_{\alpha}$.

We next investigate the infinitesimal generator of $U(t), t>0$. Define $B: C_{\alpha} \rightarrow C_{\alpha}$ as follows:

$$
\begin{gathered}
(B \phi)(\theta)=\dot{\phi}(\theta), \quad-r<\theta<0, \\
D(B)=\left\{\phi \in C_{\alpha}: \dot{\phi} \in C_{\alpha}, \phi(0) \in D(A), \dot{\phi}^{-}(0)=-A \phi(0)+F(\phi)\right\} .
\end{gathered}
$$

Notice that $\phi \in D(B)$ implies that $-A \phi(0)+F(\phi) \in D\left(A^{\alpha}\right)$.

Proposition 4.2. $B$ is the infinitesimal generator of $U(t), t>0$.

Proof. Let $\hat{B}$ denote the infinitesimal generator of $U(t), t>0$. We, of course, must show that $B=\hat{B}$ and $D(B)=D(\hat{B})$. Since the norm of $X_{\alpha}$ dominates the norm of $X$,

$$
(\hat{B} \phi)(\theta)=X-\lim _{t \rightarrow 0^{+}} \frac{(U(t) \phi)(\theta)-\phi(\theta)}{t}
$$

for all $\phi \in D(\hat{B})$ and $\theta \in[-r, 0]$. As in Proposition 3.2 of [1], we conclude that $(\hat{B} \phi)(\theta)=\dot{\phi}(\theta)$ for all $\phi \in[-r, 0]$, and that $\dot{\phi} \in C_{\alpha}$. Since

and

$$
X-\lim _{t \rightarrow 0^{+}} \frac{1}{t} \int_{0}^{t} T(t-s) F\left(u_{s}(\phi)\right) d s=F(\phi)
$$

$$
\begin{aligned}
\frac{(U(t) \phi)(0)-\phi(0)}{t} & -\frac{1}{t} \int_{0}^{t} T(t-s) F\left(u_{s}(\phi)\right) d s \\
= & \frac{T(t) \phi(0)-\phi(0)}{t},
\end{aligned}
$$

we conclude that $\phi(0) \in D(A)$ and $\dot{\phi}^{-}(0)=-A \phi(0)+F(\phi)$. Thus we have demonstrated that $D(\hat{B}) \subset D(B)$ and that $\hat{B} \phi=B \phi$ for $\phi \in D(\hat{B})$. It remains to show that $D(B) \subset D(\hat{B})$. Suppose $\phi \in D(B)$; then $\phi(0) \in D(A)$ 
and $-A \phi(0)+f(\phi) \in D\left(A^{\alpha}\right)$. Since

$$
A^{\alpha} \phi(0)-A^{\alpha-1} f(\phi)=-A^{-1} A^{\alpha}(-A \phi(0)+f(\phi)) \text {, }
$$

we have that $A^{\alpha} \phi(0)-A^{\alpha-1} f(\phi) \in D(A)$ and $-A\left(A^{\alpha} \phi(0)-A^{\alpha-1} f(\phi)\right)=$ $A^{\alpha}(-A \phi(0)+f(\phi))$.

We now show that there exists a constant $M_{\phi}$, dependent only on $\phi$, such that

$$
\left\|u_{t}(\phi)-\phi\right\|_{C_{\alpha}} \leqslant M_{\phi} t
$$

for small positive $t$. Suppose that $-r<t+\theta<0$. Then

$$
\begin{aligned}
\|u(\phi)(t+\theta)-\phi(\theta)\|_{\alpha} & =\left\|A^{\alpha} \phi(t+\theta)-A^{\alpha} \phi(\theta)\right\|=\left\|\int_{0}^{t+\theta} A^{\alpha} \dot{\phi}(s) d s\right\| \\
& <t \sup _{\theta<s<t+\theta}\|\dot{\phi}(s)\|_{\alpha} \leqslant t\|\dot{\phi}\|_{c_{\alpha} .}
\end{aligned}
$$

If $0<t+\theta$, then

$$
\begin{aligned}
\| u(\phi) & (t+\theta)-\phi(\theta) \|_{\alpha} \\
= & \left\|T(t+\theta) A^{\alpha} \phi(0)-A^{\alpha} \phi(\theta)+\int_{0}^{t+\theta} A^{\alpha} T(t+\theta-s) F\left(u_{s}(\phi)\right) d s\right\| \\
= & \|(T(t+\theta)-I)\left(A^{\alpha} \phi(0)-A^{\alpha-1} F(\phi)\right)+(T(t+\theta)-I) A^{\alpha-1} F(\phi) \\
& \quad+A^{\alpha} \phi(0)-A^{\alpha} \phi(\theta)+\int_{0}^{t+\theta} A^{\alpha} T(t+\theta-s) F\left(u_{s}(\phi)\right) d s \| \\
= & \| \int_{0}^{t+\theta}-A T(s)\left(A^{\alpha} \phi(0)-A^{\alpha-1} F(\phi)\right) d s+\int_{\theta}^{0} A^{\alpha} \dot{\phi}(s) d s \\
& \quad+\int_{0}^{t+\theta} A^{\alpha} T(t+\theta-s)\left[F\left(u_{s}(\phi)\right)-F(\phi)\right] d s \| \\
\leqslant & M \max \left\{1, e^{\omega t}\right\} t\left\|-A\left(A^{\alpha} \phi(0)-A^{\alpha-1} F(\phi)\right)\right\|+t\|\dot{\phi}\|_{c_{\alpha}} \\
& +\int_{0}^{t+\theta} M L e^{\omega(t+\theta-s)}(t+\theta-s)^{-\alpha}\left\|u_{s}(\phi)-\phi\right\|_{C_{\alpha}} d s .
\end{aligned}
$$

Thus for $0<t+\theta$ and $0<t \leqslant 1$, there exist real constants $R>0$ and $S>0$, depending only on $\phi$, such that

$$
\|u(\phi)(t+\theta)-\phi(\theta)\|_{\alpha} \leqslant R t+S t^{1-\alpha} \sup _{0<s<t}\left\|u_{s}(\phi)-\phi\right\|_{C_{a}}
$$

Then, for $0 \leqslant t \leqslant t_{1} \leqslant 1$,

$$
\left\|u_{t}(\phi)-\phi\right\|_{C_{\alpha}} \leqslant \max \left\{R,\|\dot{\phi}\|_{C_{\alpha}}\right\} t_{1}+S t_{1}^{1-\alpha} \sup _{0<s<t_{1}}\left\|u_{s}(\phi)-\phi\right\|_{C_{\alpha}} .
$$

This implies that for $t_{1}$ sufficiently small, 


$$
\sup _{0<t<t_{1}}\left\|u_{t}(\phi)-\phi\right\|_{C_{\alpha}} \leqslant t_{1} \max \left\{R,\|\dot{\phi}\|_{C_{\alpha}}\right\}\left(1-S t_{1}^{1-\alpha}\right)^{-1},
$$

from which the truth of equation (4.5) now follows.

We are now ready to show that if $\phi \in D(B)$, then $C_{\alpha}-$ $\lim _{t \rightarrow 0^{+}}(1 / t)(U(t) \phi-\phi)$ exists and is equal to $\dot{\phi}$; that is, we will show that $\phi \in D(\hat{B})$. We leave the case $-r \leqslant t+\theta \leqslant 0$ to the reader. Suppose that $0<t+\theta$, then

$$
\begin{aligned}
& \|(1 / t)[(U(t) \phi)(\theta)-\phi(\theta)]-\dot{\phi}(\theta)\|_{\alpha} \\
& =\|(1 / t)\left(T(t+\theta) A^{\alpha} \phi(0)-A^{\alpha} \phi(\theta)\right) \\
& +\frac{1}{t} \int_{0}^{t+\theta} A^{\alpha} T(t+\theta-s) F\left(u_{s}(\phi)\right) d s-A^{\alpha} \dot{\phi}(\theta) \| \\
& =\|(1 / t)(T(t+\theta)-I)\left(A^{\alpha} \phi(0)-A^{\alpha-1} F(\phi)\right) \\
& +(1 / t)(T(t+\theta)-I) A^{\alpha-1} F(\phi) \\
& -(t+\theta)(1 / t) A^{\alpha} \dot{\phi}(0)+\frac{1}{t} \int_{0}^{t+\theta} A^{\alpha} T(t+\theta-s) F\left(u_{s}(\phi)\right) d s \\
& +(1 / t)\left(A^{\alpha} \phi(0)-A^{\alpha} \phi(\theta)\right)+(t+\theta)(1 / t) A^{\alpha} \dot{\phi}(0)-A^{\alpha} \dot{\phi}(\theta) \| \\
& <\left\|\frac{1}{t} \int_{0}^{t+\theta} T(s) A^{\alpha}(-A \phi(0)+F(\phi))-A^{\alpha}(-A \phi(0)+F(\phi)) d s\right\| \\
& +\left\|\frac{1}{t} \int_{0}^{t+\theta} A^{\alpha} T(t+\theta-s)\left[F\left(u_{s}(\phi)\right)-F(\phi)\right] d s\right\| \\
& +\left\|\frac{1}{t} \int_{\theta}^{0}\left[A^{\alpha} \dot{\phi}(s)-A^{\alpha} \dot{\phi}(0)\right] d s\right\|+\left\|A^{\alpha} \dot{\phi}(0)-A^{\alpha} \dot{\phi}(\theta)\right\| \\
& <\sup _{0<s<t}\left\|(T(s)-I) A^{\alpha}(-A \phi(0)+F(\phi))\right\| \\
& +\frac{1}{t} \int_{0}^{t+\theta} M L e^{\omega(t+\theta-s)}(t+\theta-s)^{-\alpha} d s \sup _{0<s<t}\left\|u_{s}(\phi)-\phi\right\|_{C_{\varepsilon}} \\
& +2 \sup _{\theta<s<0}\left\|A^{\alpha} \dot{\phi}(s)-A^{\alpha} \dot{\phi}(0)\right\| \\
& <\sup _{0<s<t}\left\|(T(s)-I) A^{\alpha}(-A \phi(0)+F(\phi))\right\| \\
& +\frac{1}{t} M L \max \left\{1, e^{\omega t}\right\} \frac{(t+\theta)}{1-\alpha}^{1-\alpha} M_{\phi} t+2 \sup _{\theta<s<0}\left\|A^{\alpha} \dot{\phi}(s)-A^{\alpha} \dot{\phi}(0)\right\| \text {. }
\end{aligned}
$$


The truth of Proposition 4.2 now follows.

5. Stability in the autonomous linear case. In this section, $F$ will be as in $\S 4$ except that we require $F$ to be linear. For each $\lambda \in \mathbf{C}$, define the linear operator $\Delta(\lambda): X_{\alpha} \rightarrow X_{\alpha}$ by

$$
\begin{gathered}
\Delta(\lambda) x=-A x-\lambda x+F\left(e^{\lambda \theta} x\right) \\
D(\Delta(\lambda))=\left\{x \in X_{\alpha}: x \in D(A),-A x+F\left(e^{\lambda \theta} x\right) \in D\left(A^{\alpha}\right)\right\} .
\end{gathered}
$$

If $\Delta(\lambda) x=0$ for some nonzero $x \in D(\Delta(\lambda))$, we say that $\lambda$ is a characteristic value. With the aid of Proposition 4.1, the following results concerning stability may be established as in Proposition 4.1 of [1].

Proposition 5.1. (i) $\lambda$ is an eigenvalue of $B$ iff $\Delta(\lambda) x=0$ for some nonzero $x \in D(\Delta(\lambda))$.

(ii) There exists a real number $\beta$ such that if $\lambda$ is a characteristic value, then $\operatorname{Re} \lambda \leqslant \beta$.

(iii) If $\beta$ is a real number satisfying (ii), then for every $\gamma>0$, there exists $a$ constant $K(\gamma)>1$ such that for all $t \geq 0, \phi \in C_{\alpha}$,

$$
\|U(t) \phi\|_{C_{\alpha}} \leqslant K(\gamma) e^{(\beta+\gamma) t}\|\phi\|_{C_{\alpha}} .
$$

Proposition 5.2. Let $\beta$ be the smallest real number such that if $\lambda$ is a characteristic value, then $\operatorname{Re} \lambda \leqslant \beta$. If $\beta<0$, then for all $\phi \in C_{\alpha},\|U(t) \phi\|_{C_{\alpha}}$ $\rightarrow 0$ as $t \rightarrow \infty$. If $\beta=0$, then there exists $\phi \neq 0 \in C_{\alpha}$ such that $\|U(t) \phi\|_{C_{\alpha}}=$ $\|\phi\|_{C_{a}}$ for all $t \geqslant 0$. If $\beta>0$, then there exists $\phi \in C_{\alpha}$ such that $\|U(t) \phi\|_{C_{\alpha}} \rightarrow$ $\infty$ as $t \rightarrow \infty$.

EXAMPLE 5.1. To illustrate the theory we have developed, we shall demonstrate that equations of the form

$$
\begin{aligned}
\frac{\partial W}{\partial t}(x, t) & =\frac{\partial^{2} W}{\partial x^{2}}(x, t)+f\left(W(x, t-r), \frac{\partial W}{\partial x}(x, t-r)\right), \\
W(0, t) & =W(\pi, t)=0, \quad t \geqslant 0, \quad 0 \leqslant x \leqslant \pi, \quad t \geqslant \\
W(x, t) & =\phi(x, t), \quad 0 \leqslant x \leqslant \pi, \quad-r \leqslant t \leqslant 0,
\end{aligned}
$$

where $f: R \times R \rightarrow R, f(0,0)=0, f$ is continuous in its first variable and Lipschitz continuous in its second variable satisfy the assumptions (2.2)-(2.7).

Let $X=L^{2}[0, \pi]$ and let $A: X \rightarrow X$ be defined by

$$
A Y=-Y^{\prime \prime} \text {, }
$$

$D(A)=\left\{Y \in X: Y, Y^{\prime}\right.$ are absolutely continuous,

$$
\left.Y^{\prime \prime} \in X, Y(0)=Y(\pi)=0\right\}
$$


Then

$$
A Y=\sum_{n=1}^{\infty} n^{2}\left(Y, Z_{n}\right) Z_{n}, \quad Y \in D(A),
$$

where $Z_{n}(s)=\sqrt{2 / \pi} \sin n s, n=1,2, \ldots$, is the orthonormal set of eigenvectors of $A$. It is well known that $-A$ is the infinitesimal generator of an analytic semigroup $T(t), t \geqslant 0$, in $X$ given by

$$
T(t) Y=\sum_{n=1}^{\infty} \exp \left(-n^{2} t\right)\left(Y, Z_{n}\right) Z_{n}, \quad Y \in X
$$

$T(t), t>0$, satisfies the inequality in (2.2) with $M>1$ and $\omega>-1$.

If we choose $\alpha=\frac{1}{2}$, then $A$ satisfies (2.3) with $-1<\omega<0, M_{1 / 2}=$ $1 /(2 e(1+\omega))^{1 / 2}$, since

$$
A^{1 / 2} T(t) Y=\sum_{n=1}^{\infty} n \exp \left(-n^{2} t\right)\left(Y, Z_{n}\right) Z_{n}, \quad Y \in X,
$$

and

$$
\left\|A^{1 / 2} T(t) Y\right\|^{2}<\sup _{n>1}\left\{n^{2} \exp \left(-2 n^{2} t\right)\right\}\|Y\|^{2}, \quad Y \in X,
$$

and $t n^{2} \exp \left[-2 t\left(n^{2}+\omega\right)\right]<1 / 2 e(1+\omega)$ for $n=1,2, \ldots$ In addition, (2.4) is satisfied for $\alpha=\frac{1}{2}$, since

$$
A^{-1 / 2} Y=\sum_{n=1}^{\infty}(1 / n)\left(Y, Z_{n}\right) Z_{n}, \quad Y \in X,
$$

and

$$
A^{-1 / 2} T(t) Y=\sum_{n=1}^{\infty}(1 / n) \exp \left(-n^{2} t\right)\left(Y, Z_{n}\right) Z_{n}, \quad Y \in X,
$$

and thus

$$
\left\|(T(t)-I) A^{-1 / 2} Y\right\|^{2} \leqslant \sup _{n>1}\left\{\left(1 / n^{2}\right)\left(\exp \left(-n^{2} t\right)-1\right)^{2}\right\}\|Y\|^{2}
$$

Since $\left(\exp \left(-n^{2} t\right)-1\right)^{2} / n^{2}<t / 2$ for $n=1,2, \ldots$, we may choose $N_{1 / 2}=1 / \sqrt{2}$.

$T(t), t>0$, satisfies (2.5) since an analytic semigroup is compact for each $t>0$ if and only if $A^{-1 / 2}$ is compact. The compactness of $A^{-1 / 2}$ follows from the fact that the eigenvalues of $A^{-1 / 2}$ are $\lambda_{n}=1 / n, n=1,2, \ldots$

Let $F: C_{1 / 2} \rightarrow X$ be defined by

$$
F(\phi)(s)=f\left(\phi(-r)(s), \phi(r)^{\prime}(s)\right), \quad \phi \in C_{1 / 2}, s \in[0, \pi] .
$$

That $F$ is well defined follows from the fact that $f$ is continuous in the first variable, Lipschitz continuous in the second variable, and that $Y \in D\left(A^{1 / 2}\right)$ implies $Y$ is absolutely continuous and $Y^{\prime} \in X$. F satisfies (2.7), since for $\phi, \psi \in C_{1 / 2}$, 


$$
\begin{aligned}
\| \phi(-r)^{\prime} & -\psi(-r)^{\prime} \|_{X}^{2}=\int_{0}^{\pi}\left|\phi(-r)^{\prime}(s)-\psi(-r)^{\prime}(s)\right|^{2} d s \\
& =\sum_{n=1}^{\infty} \sum_{m=1}^{\infty}\left(\phi(-r)-\psi(-r), Z_{n}\right)\left(\phi(-r)-\psi(-r), Z_{m}\right)\left(Z_{n}^{\prime}, Z_{m}^{\prime}\right) \\
& =\sum_{n=1}^{\infty} \sum_{m=1}^{\infty}\left(\phi(-r)-\psi(-r), Z_{n}\right)\left(\phi(-r)-\psi(-r), Z_{m}\right)\left(-Z_{n}^{\prime \prime}, Z_{m}\right) \\
& =\sum_{n=1}^{\infty}\left(\phi(-r)-\psi(-r), Z_{n}\right)^{2} n^{2}=\left\|A^{1 / 2}(\phi(-r)-\psi(-r))\right\|^{2} \\
& <\|\phi-\psi\|_{C_{1 / 2}}^{2} .
\end{aligned}
$$

and

$$
\begin{aligned}
\|\phi(-r)-\psi(-r)\|_{X}^{2}=\int_{0}^{\pi}|\phi(-r)(s)-\psi(-r)(s)|^{2} d s \\
\quad=\sum_{n=1}^{\infty}\left(\phi(-r)-\psi(-r), Z_{n}\right)^{2}<\sum_{n=1}^{\infty} n^{2}\left(\phi(-r)-\psi(-r), Z_{n}\right)^{2} \\
<\left\|A^{1 / 2}(\phi(-r)-\psi(-r))\right\|^{2}<\| \phi-\psi_{C_{1 / 2}}^{2}
\end{aligned}
$$

Thus the mapping from $C_{1 / 2} \rightarrow X \times X$ defined by $\phi \rightarrow\left(\phi(-r), \phi(-r)^{\prime}\right)$ is continuous. The continuity of $F$ now follows.

We have now demonstrated that equations of form (5.2), where $f$ is continuous in the first variable and Lipschitz continuous in the second variable, satisfy assumptions (2.2)-(2.7). As a final application of the theory we have developed, we will determine necessary and sufficient conditions for the asymptotic stability of two particular equations of form (5.2) in the case where $f$ is linear.

EXAMPLE 5.2. We wish to determine the exact region of stability of the linear equation

$$
\begin{aligned}
\frac{\partial W}{\partial t}(x, t) & =\frac{\partial^{2} W}{\partial x^{2}}(x, t)-a \frac{\partial W}{\partial x}(x, t)-b W(x, t-r), \\
W(0, t) & =W(\pi, t)=0, \quad t>0, \\
W(x, t) & =\phi(x, t), \quad 0<x<\pi, \quad-r<t<0 .
\end{aligned}
$$

Equation (5.4) can be written in the form (1.1) where $A$ is defined by (5.3) and $F: C_{1 / 2} \rightarrow X$ is given by $F(\phi)=-a \phi(0)^{\prime}-b \phi(-r)$. The characteristic equation is then given by

$$
\Delta(\lambda) f=-A f-\lambda f-a f^{\prime}-b e^{-\lambda r},
$$

where $f \neq 0, f \in D(A)$, and $-A f-a f^{\prime}-b e^{-\lambda r_{f}} \in D\left(A^{1 / 2}\right)$. By Proposition 5.2 , the zero solution of equation (5.4) is asymptotically stable if and only if $\beta<0$, where $\beta$ is the smallest real number such that if $\lambda$ is a characteristic 
value, then $\operatorname{Re} \lambda \leqslant \beta$. Since the eigenvalues of the operator $M: L^{2}[0, \pi] \rightarrow$ $L^{2}[0, \pi]$ defined by $M f=f^{\prime \prime}-a f^{\prime}, D(M)=\left\{f \in L^{2}[0, \pi]: f, f^{\prime}\right.$ are absolutely continuous, $\left.f^{\prime \prime} \in L^{2}[0, \pi], f(0)=f(\pi)=0\right\}$ are given by $\lambda_{n}=-n^{2}-$ $a^{2} / 4, n=1,2, \ldots$, we have from (5.5) that the zero solution of equation (5.4) is asymptotically stable if and only if all of the roots of the equations

$$
\lambda+a^{2} / 4+b e^{-\lambda r}=-n^{2}, \quad n=1,2, \ldots,
$$

have negative real parts. The exact region of stability of equation (5.4) as a function of $a, b$, and $r$ is indicated in Figure $\mathrm{I}$.

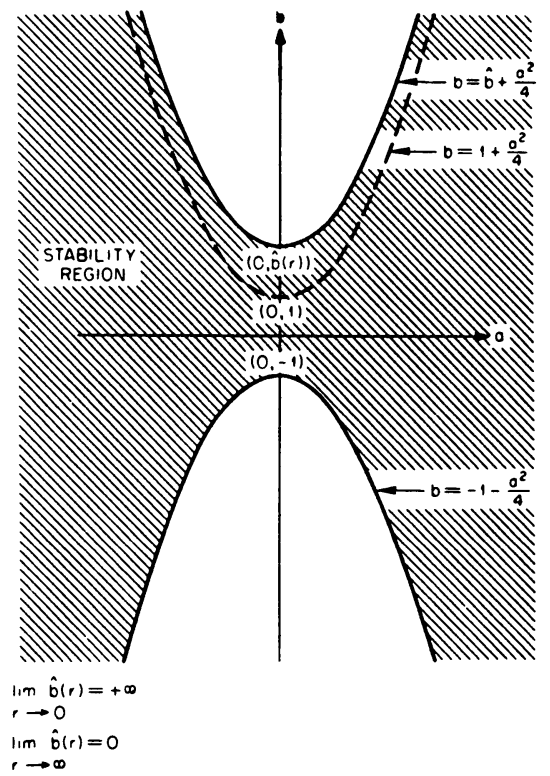

FIGURE I

EXAMPLE 5.3. Consider the equation

$$
\begin{aligned}
\frac{\partial W}{\partial t}(x, t) & =\frac{\partial^{2} W}{\partial x^{2}}(x, t)-a \frac{\partial W}{\partial x}\left(x, t-\frac{r}{2}\right)-b W(x, t-r), \\
W(0, t) & =W(\pi, t)=0, \quad t \geqslant 0, \\
W(x, t) & =\phi(x, t), \quad 0<x \leqslant \pi, \quad-r \leqslant t \leqslant 0 .
\end{aligned}
$$

Let $X=L^{2}[0, \pi]$ and $A: X \rightarrow X$ be defined by (5.3). Then equation (5.6) can be written in the form (1.1) where $F: C_{1 / 2} \rightarrow X$ is given by $F(\phi)=$ $-a \phi(-r / 2)^{\prime}-b \phi(-r)$. The characteristic equation is then

$$
\Delta(\lambda) f=-A f-\lambda f-a e^{-\lambda r / 2} f^{\prime}-b e^{-\lambda r} f,
$$

where $f \neq 0, f \in D(A)$, and $-A f-a e^{-\lambda r / 2 f^{\prime}}-b e^{-\lambda_{r} f} \in D\left(A^{1 / 2}\right)$. It now follows from Proposition 5.2 that the zero solution of equation (5.6) is 
asymptotically stable if and only if all of the roots of the family of transcendental equations

$$
\lambda+a^{2} e^{-\lambda r} / 4+b e^{-\lambda r}=-n^{2}, \quad n=1,2, \ldots,
$$

have negative real parts. The exact region of stability of equation (5.6) as a function of $a, b$, and $r$ is given in Figure II.

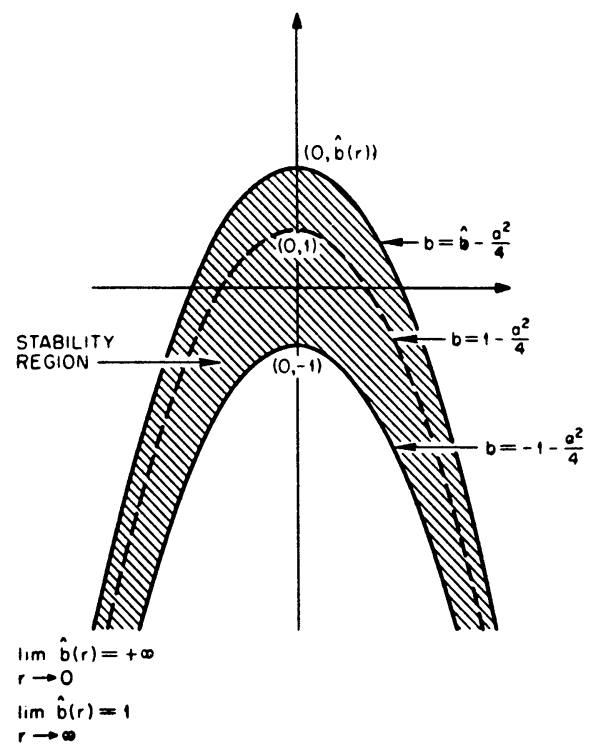

FIGURE II

\section{REFERENCES}

1. C. C. Travis and G. F. Webb, Existence and stability for partial functional differential equations, Trans. Amer. Math. Soc. 200 (1974), 395-418.

2. $\longrightarrow$ Partial differential equations with deviating arguments in the time variable, J. Math. Anal. Appl. 56 (1976), 397-409.

3. D. Henry, Geometric theory of nonlinear parabolic equations (to appear).

Department of Mathematics, University of Tennessee, Knoxville, Tennessee 37916

Department of Mathematics, Vanderbitt University, Nashville, Tennessee 37203 (Current address of G. F. Webb)

Current address (C. C. Travis): Oak Ridge National Laboratory, Post Office Box X, Oak Ridge, Tennessee 37830 